\section{Gonalgia entre trabalhadores e fatores ocupacionais associados: uma revisão sistemática}

\author{
Knee pain and associated occupational factors: \\ a systematic review
}

\footnotetext{
1 Programa de Pós-graduação em Epidemiologia,

Universidade Federal de

Pelotas, Pelotas, Brasil.

2 Universidade do Vale do

Rio dos Sinos, São Leopoldo,

Brasil

3 Department of Work

Environment, University of

Massachusetts, Lowell, U.S.A.

Correspondência

M. C. Silva

Programa de Pós-graduação

em Epidemiologia,

Universidade Federal de

Pelotas.

Av. Duque de Caxias 250,

Pelotas, RS

96030-002, Brasil.

cozzensa@terra.com.br
}

\begin{abstract}
In order to collect information on knee pain and associated occupational factors, a systematic review was conducted using the MEDLINE, LILACS, SciELO, and Free Medical Journals databases, from 1990 to 2006. Key words were: gonalgia, knee, knee pain, knee joint, knee dislocation, knee injuries, work, workplace, workload, employment, occupations, industry, occupational, workers, arthrosis, and osteoarthritis. Equivalent terms in Portuguese and Spanish were also used. From the initial 2,263 studies gathered, only 26 met the review's inclusion criteria. Knee pain prevalence in the previous 12 months ranged from $11.2 \%$ to $60.9 \%$. The main associated factors were: female gender, older age, high body mass index, kneeling working position, and lifting at work.
\end{abstract}

Musculoskeletal Diseases; Occupational Health; Review Literature
Marcelo Cozzensa da Silva 1 Anaclaudia Gastal Fassa 1 Marlos Rodrigues Domingues 1,2 David Kriebel 3

\section{Introdução}

As afecções musculoesqueléticas, principalmente as lombalgias e algias dos membros superiores, ocupam os primeiros lugares entre as doenças crônico-degenerativas no que diz respeito ao perfil de morbidade em diversos países 1 . O custo anual com desordens musculoesqueléticas tem sido estimado entre 1,0 e $2,5 \%$ do produto nacional bruto de países como Estados Unidos, Inglaterra, França e Austrália ${ }^{2}$. Nos Estados Unidos, o National Institute for Occupational Safety and Health (NIOSH) 3, em 1997, classificou essas afecções, principalmente as da coluna, como a segunda causa mais importante de afastamento temporário do trabalho no país. Dados do Departamento do Trabalho desse país indicam que, ao considerar os acidentes e as doenças ocupacionais dos trabalhadores de construção, os problemas musculoesqueléticos apresentam a segunda maior incidência ${ }^{4}$. No Brasil, segundo os dados de 1986, do Instituto Nacional de Previdência Social (INPS), as doenças do sistema musculoesquelético figuram como a terceira causa de aposentadorias por invalidez e a primeira causa de auxílio-doença ${ }^{5}$.

As gonalgias, popularmente conhecidas como dor nos joelhos, apesar de menos prevalentes e menos estudadas, também constituem um problema de saúde pública que possivelmente gera um enorme custo para o sistema de saúde. Entretanto, estimativas diretas desses gastos ainda 
são escassas 6. Em 1996-1997, mais de 6 milhões de americanos procuraram os serviços de saúde por doenças relacionadas aos joelhos, sendo que, destes, 5 milhões visitaram consultórios de cirurgiões ortopedistas e 1,4 milhão foi para as emergências hospitalares 7 . Um estudo realizado pelos cirurgiões ortopédicos dos Estados Unidos em 1997 revelou que o joelho era a região anatômica mais tratada, sendo responsável por $26 \%$ do total de todas as visitas a ortopedistas 7 . A prevalência desse tipo de morbidade varia de 10 a $60 \%$ dependendo da idade, ocupação e definição de gonalgia 8,9 , mas estas estimativas provêm dos poucos estudos epidemiológicos que existem sobre o tema e seus fatores de risco no trabalho.

A maioria das pesquisas atuais procura utilizar-se de diagnósticos radiográficos para a verificação de osteoartrite. O grande problema desse método é que a gonalgia freqüentemente ocorre sem a presença de osteoartrite, que é um doença altamente relacionada com a idade 10,11,12. Gonalgia, entretanto, não ocorre somente com o avanço da idade ou com o aparecimento de osteoartrite, mas também pode estar associada a fatores sócio-econômicos 8,13 e lesões prévias de joelho 14 . Desse modo, osteoartrite e dor no joelho podem ser determinadas por fatores de risco diferentes.

O conhecimento sobre os fatores de risco para as dores de joelho, particularmente com relação à carga de trabalho ocupacional é, ainda, limitado, principalmente devido ao pequeno número de estudos prospectivos 15. Estudo transversal mostrou que a prevalência de gonalgia aumentou com a idade e foi superior entre os indivíduos do sexo feminino 16,17. Menor nível educacional tem se mostrado associado à gonalgia em várias pesquisas 8,13. Em relação às ocupações, estudos mostram que indivíduos que trabalham em profissões que exigem maior demanda física, tais como a de carpintaria, construção, mineração e outros, apresentam maior prevalência de gonalgia quando comparados a trabalhadores com demandas físicas menores 9. Estudo realizado por Bergenudd et al. 8 demonstrou que ocupações com moderadas cargas de trabalho apresentavam maior risco de gonalgia do que aqueles com cargas leves ou pesadas.

Informações sobre a distribuição dessa morbidade por tipo de ocupação são necessárias para se estimar a sobrecarga das desordens nos joelhos relacionadas ao trabalho. Sendo assim, é importante avaliar o impacto desse problema por meio de uma busca ativa na literatura que reúna o conhecimento produzido até o momento e descreva melhor esta realidade.

O objetivo deste estudo é o de apresentar uma revisão sistemática dos estudos epidemiológicos existentes sobre gonalgia em trabalhadores, realizados a partir do ano de 1990. Examinar-se-á as prevalências e os fatores de risco para dor no joelho, bem como as vantagens e limitações das abordagens utilizadas.

\section{Metodologia}

Foram incluídos na revisão artigos indexados, publicados desde 1990, escritos em inglês, português e espanhol, que avaliaram gonalgia como desfecho principal ou secundário em trabalhadores. Foram aceitos artigos com trabalhadores do mercado formal ou informal, sem qualquer restrição de idade, sexo e etnia ou quanto ao tipo de estudo. Artigos que apresentavam osteoartrite como principal alvo de estudo, mas que também possuíam informações sobre gonalgias, foram incluídos na análise.

A estratégia de busca de artigos incluiu pesquisa em bases eletrônicas e busca manual de citações nas publicações inicialmente identificadas. Utilizaram-se as bases eletrônicas MEDLINE (National Library of Medicine, Estados Unidos), LILACS (Literatura Latino-Americana e do Caribe em Ciências da Saúde), SciELO (Scientific Eletronic Library Online), biblioteca eletrônica da Universidade de Massachusetts Lowell (Estados Unidos) e o site Free Medical Journals (http:/ / www.freemedicaljournal.com). A busca manual foi realizada nas bibliotecas da Universidade de Massachusetts Lowell; da Faculdade de Medicina, Universidade Federal de Pelotas (UFPEL) e do Programa de Pós-graduação em Epidemiologia, UFPEL. Além disso, contato direto com alguns autores via $e$-mail viabilizou a obtenção de vários artigos.

Os descritores utilizados em inglês foram: knee, knee pain, knee joint, knee dislocation, knee injuries, work, workplace, workload, employment, occupations, industry, occupational, workers, arthrosis, osteoarthritis e myositis, bem como seus correspondentes em português e espanhol.

Os artigos selecionados foram avaliados e pontuados por dois epidemiologistas conforme uma adaptação dos critérios de Downs \& Black 18 , para estudos observacionais. Os itens relacionados apenas a estudos de intervenção foram excluídos, pois nenhuma publicação revisada era do tipo experimental. Sendo assim, analisaram-se os artigos com base na: (1) qualidade da descrição de hipóteses/objetivos; (2) qualidade da descrição do desfecho a ser estudado; (3) caracterização da amostra incluída; (4) qualidade da descrição e discussão dos principais fatores de confusão; (5) qualidade da descrição dos principais achados do estudo; (6) inclusão 
dos principais valores de probabilidade para os principais desfechos; (7) representatividade da amostra em relação à população em estudo; (8) indicação de que os resultados não tenham sido baseados em hipóteses a priori, quando este fosse o caso; (9) apropriação dos testes estatísticos utilizados para medir os principais desfechos; (10) acurácia dos instrumentos utilizados para os principais desfechos; (11) adequação do ajuste para os principais fatores de confusão; (12) adequação do poder estatístico para detectar um efeito importante, com um nível de significância de $5 \%$.

O total de itens avaliados segundo o critério adaptado de Downs \& Black 18 foi de 12, pontuando, no máximo, 13 pontos (todos os itens valem um ponto, com exceção do item 4 que tem pontuação máxima de dois).

\section{Resultados}

Foram encontrados 2.263 títulos nas bases de dados on-line. O passo inicial foi o de eliminar os artigos repetidos. Foram encontrados e excluídos 145 artigos escritos em línguas diferentes do inglês, português e espanhol. A seguir foram eliminados os artigos que não apresentavam abstract, artigos utilizando metodologias qualitativas, artigos em atletas profissionais e amadores (os quais já se conhece a relação entre a sobrecarga intensa da atividade e gonalgia) e artigos que não tratavam de saúde ocupacional ou não avaliavam variáveis ocupacionais. Ao final desse processo permaneceram 204 artigos em nossa base de dados. Desses, foram eliminados os que não apresentavam pelo menos uma das informações: prevalência/incidência de gonalgia ou análise da associação entre gonalgia e fatores ocupacionais. Então, chegamos ao final da busca com 26 artigos nesta revisão.

O escore metodológico foi, em média, de 9,7 pontos ( $\mathrm{dp}=2,01$ pontos). O estudo que obteve maior pontuação (13) foi o de Fassa et al. 19. Quarenta e dois por cento dos artigos tiveram pontuação igual ou inferior a oito, sendo que a menor pontuação obtida foi sete 9,20,21,22,23. Dentre os critérios de Downs \& Black 18 , as principais limitações dos estudos foram a falta de representatividade amostral e o baixo poder estatístico para detectar efeitos importantes. A pontuação de cada um dos estudos encontra-se na Tabela 1.

Dentre os estudos selecionados, 12 foram conduzidos em países europeus 9,15,21,23,24,25,26,27, 28,29,30,31, sete na América do Norte 3,20,32,33,34,35,36, quatro na América do Sul 19,22,37,38 e três na Ásia 6,39,40. A maioria dos estudos incluiu indivíduos de ambos os sexos (62\%), no entanto, sete con- tavam apenas com pessoas do sexo masculino $21,25,26,27,28,34,39$ e três com pessoas do sexo feminino $29,38,40$. O estudo de Fassa et al. ${ }^{19}$, com crianças trabalhadoras, foi o que apresentou a menor média de idade amostral (13,0 anos; $\mathrm{dp}=2,3$ anos), e o de Forde et al. 33 , com trabalhadores da construção, a maior (49,6 anos; dp = 13,7 anos).

Os trabalhadores da construção $3,25,33,34$, os instaladores de piso $21,26,27,28$ e os enfermeiros 29,36,38,40 foram as categorias de trabalho mais estudadas, cada qual com quatro artigos, seguidas pelos trabalhadores da indústria 15,30, com dois. Também foram encontrados estudos com taxistas, bombeiros, trabalhadores rurais, militares, eletricistas, bancários, carpinteiros, comerciários, catadores de materiais recicláveis e crianças trabalhadoras. Um artigo de base populacional e outro que abordou categorias diversas de trabalho também foram analisados (Tabela 1).

Do total de artigos utilizados, apenas três foram de coorte 15,23,35, sendo que todos os demais apresentaram delineamento transversal. O instrumento mais utilizado para a verificação da gonalgia foi o Nordic Musculoskeletal Questionnaire 41 . Foram 13 os estudos utilizando o instrumento original $6,19,21,26,27,29,37,38$ ou uma versão modificada do mesmo 3,15,22,30,40. O estudo de Forde et al. 33 e o de Nahit et al. 31 utilizaram uma figura semelhante à preconizada por Kuorinka et al. ${ }^{41}$ para a verificação da gonalgia. Cornell Musculoskeletal Disconfort Questionnaire foi utilizado por Menzel et al. ${ }^{36}$ para a verificação do mesmo desfecho. Os estudos de Gomez et al. ${ }^{35}$, O’Reilly et al. ${ }^{9}$, Kivimaki et al. 28 e Goldsheyder et al. 34 utilizaram questionários com perguntas específicas sobre dor na articulação dos joelhos.

Aproximadamente $77 \%$ dos estudos caracterizaram a gonalgia nos últimos 12 meses. Destes, seis também coletaram informações sobre dor nessa articulação nos últimos 7 dias 21,22,27,30,34,38. Menzel et al. 36 somente coletaram informações sobre gonalgia nos últimos 7 dias. Kivimaki et al. 28 e Nahit et al. 31 caracterizaram a prevalência de gonalgia por meio da sintomatologia de dor ou desconforto nessa região no mês anterior à aplicação do questionário.

A maioria dos estudos foi direcionada a uma categoria específica de trabalhadores e, nestes casos, a análise das associações incluiu uma série de variáveis relacionadas aos esforços realizados e à ergonomia de trabalho, tais como posições e cargas de trabalho.

A análise dos dados consistiu na descrição dos resultados e de testes de associação entre os fatores de risco em estudo e a variável dependente. Do total de artigos revisados, onze 19,22,23,25,28, $33,34,37,38,39,40$ não tinham gonalgia como desfe- 
cho principal ou não realizaram testes de associação com outras variáveis coletadas nos estudos, apresentando somente resultados de prevalência. A maioria dos estudos que realizaram análise multivariável utilizou regressão logística, estimando o odds ratio como medida de efeito 4,6,9,15,24,26,27,29,30,31,35. Menzel et al. 36 utilizaram regressão linear para estimar a medida de efeito, e da Silva et al. 37 usaram regressão de Poisson.

A maior e menor prevalência de gonalgia nos últimos 7 dias foram nos instaladores de piso/ carpete e nos designers gráficos, respectivamente $32,4 \%$ e $7,0 \% 27$. As prevalências de gonalgia nos últimos 12 meses variaram entre $60,9 \%$ (carpinteiros) 9 e 11,2\% (comércio) 19. Nos profissionais de enfermagem foram encontradas prevalências entre $23,5 \%$ e $33,3 \%$ 38,40, enquanto que nos trabalhadores da construção variou entre $12,1 \%$ e $52,0 \% 19,25$. Nas demais categorias de trabalho estudadas, os trabalhadores rurais e eletricistas apresentaram prevalência em torno de $30 \%$, taxistas, catadores de materiais recicláveis e bombeiros em torno de $20 \%$ e militares $9 \%$ $6,20,23,24,35,37$.

Os principais fatores de risco associados à gonalgia estão resumidos na Tabela 1. Dentre as variáveis sócio-demográficas e nutricional, destacam-se a idade avançada 29,30,35 e índices de massa corporal (IMC) relativo a sobrepeso e obesidade 15,29,30,35. A magnitude dos riscos é bastante variável entre os artigos, de 30 a $220 \%$. Para tabagismo 15 o risco foi de $30 \%$. Dentre as variáveis ocupacionais, destacam-se o trabalho ajoelhado entre os instaladores de piso; a intensidade de trabalho nos instaladores de piso; estresse no trabalho entre os taxistas, instaladores de piso e trabalhadores da indústria; anos de trabalho entre os aprendizes da construção e eletricistas, todos com riscos superiores a $80 \%$. Trabalho ajoelhado entre os catadores de materiais recicláveis apresentou risco de $50 \%$. Os instaladores de carpete expostos a trabalho agachado e os aprendizes da construção submetidos à posição estática ou viciosa tiveram também maiores riscos para gonalgia (Tabela 1).

Dos quatro artigos que avaliaram gonalgia em trabalhadores da construção, somente um 3 estudou os fatores de risco para esta morbidade. Os principais fatores de risco associados à gonalgia no estudo de Merlino et al. 4 foram: anos de trabalho na indústria (tendência positiva), posição estática por longo tempo (OR = 2,11; IC95\%:

Tabela 1

Características dos estudos que avaliaram medidas de ocorrência e/ou efeito da ocupação na gonalgia.

\begin{tabular}{|c|c|c|c|c|c|c|c|c|}
\hline Referência & $\begin{array}{c}\text { Local do } \\
\text { estudo/Ano }\end{array}$ & Delineamento & Amostra & Instrumento & $\begin{array}{c}\text { Categoria de } \\
\text { trabalho e prevalência } \\
\text { de dor joelho (\%) }\end{array}$ & $\begin{array}{l}\text { Período } \\
\text { da } \\
\text { medida }\end{array}$ & $\begin{array}{l}\text { Fatores associados } \\
\text { (medidas de efeito; } \\
\text { IC95\%) }\end{array}$ & Escore * \\
\hline Da Silva et al. 37 & Brasil/2006 & Transversal & $\begin{array}{l}881 \text { (553 homens } \\
\text { e } 328 \text { mulheres); } \\
\text { idade } 18 \text { anos ou } \\
\text { mais; média de } \\
\text { idade }=38,0 ; 8 \% \\
\text { perdas e recusas }\end{array}$ & $\begin{array}{l}\text { Nordic } \\
\text { Musculoskeletal } \\
\text { Questionnaire }\end{array}$ & $\begin{array}{c}\text { Catadores de } \\
\text { materiais recicláveis } \\
(19,7) ; \text { Serviço não } \\
\text { doméstico }(19,0) ; \\
\text { Serviço doméstico } \\
(26,8) ; \text { Comércio } \\
(21,9) ; \text { Indústria }(17,4) ; \\
\text { Construção }(20,3) ; \\
\text { Outros }(26,3)\end{array}$ & 12 meses & $\begin{array}{c}\text { Sexo feminino } \\
(\mathrm{RP}=1,5 ; 1,3-1,7), \\
\text { idade }(\mathrm{RP}=1,2 ; \\
1,0-1,4), \text { ser casado } \\
\text { ou viver com } \\
\text { companheiro } \\
(\mathrm{RP}=1,3 ; 1,1-1,4), \\
\text { trabalhar sentado } \\
(\mathrm{RP}=0,7 ; 0,5-0,8) \text { e } \\
\text { trabalhar ajoelhado } \\
(\mathrm{RP}=1,5 ; 1,3-1,7)\end{array}$ & 12 \\
\hline Fassa et al. 19 & Brasil/2005 & Transversal & $\begin{array}{c}3.269 \text { crianças } \\
\text { ( } 1.665 \text { meninos } \\
\text { e } 1.601 \text { meninas); } \\
\text { idade } 10-17 \text { anos; } \\
7,6 \% \text { perdas e } \\
\text { recusas }\end{array}$ & $\begin{array}{l}\text { Nordic } \\
\text { Musculoskeletal } \\
\text { Questionnaire }\end{array}$ & $\begin{array}{c}\text { Serviço não } \\
\text { doméstico }(22,7) ; \\
\text { Serviço doméstico } \\
(25,5) \text {; Comércio }(11,2) ; \\
\text { Construção }(12,1) ; \\
\text { Manufatura }(19,0) ; \\
\text { Outros }(11,8)\end{array}$ & 12 meses & - & 13 \\
\hline
\end{tabular}

(continua) 


\begin{tabular}{|c|c|c|c|c|c|c|c|c|}
\hline Referência & $\begin{array}{l}\text { Local do } \\
\text { estudo/Ano }\end{array}$ & Delineamento & Amostra & Instrumento & $\begin{array}{c}\text { Categoria de } \\
\text { trabalho e prevalência } \\
\text { de dor joelho }(\%)\end{array}$ & $\begin{array}{l}\text { Período } \\
\text { da } \\
\text { medida }\end{array}$ & $\begin{array}{l}\text { Fatores associados } \\
\text { (medidas de efeito; } \\
\text { IC95\%) }\end{array}$ & Escore * \\
\hline Forde et al. 33 & $\begin{array}{c}\text { Estados } \\
\text { Unidos/2005 }\end{array}$ & Transversal & $\begin{array}{c}960 \text { homens } \\
\text { (média de idade = } \\
49,6) \text { e } 21 \text { mulheres } \\
(38,5) ; \text { idade média } \\
\text { total }=48,8 ; 37 \% \\
\text { perdas e recusas }\end{array}$ & $\begin{array}{l}\text { Questionário } \\
=\text { por telefone } \\
\text { a }\end{array}$ & $\begin{array}{l}\text { Trabalhadores da } \\
\text { construção em } \\
\text { ferro }(39,4)\end{array}$ & 12 meses & - & 8 \\
\hline Jensen 26 & $\begin{array}{c}\text { Dinamarca } \\
\text { /2005 }\end{array}$ & Transversal & $\begin{array}{l}966 \text { homens; } \\
\text { 45\% perdas e } \\
\text { recusas }\end{array}$ & $\begin{array}{c}\text { Nordic } \\
\text { Musculoskeletal } \\
\text { Questionnaire }\end{array}$ & $\begin{array}{l}\text { Instaladores de } \\
\text { carpete }(53,0)\end{array}$ & 12 meses & $\begin{array}{c}\text { Risco de gonalgia } \\
\text { aumenta positivamente } \\
\text { com o nível de intensi- } \\
\text { dade de exposição a } \\
\text { trabalho ajoelhado e } \\
\text { agachado: baixa/ } \\
\text { moderada intensidade } \\
\text { (OR }=2,9 ; 1,8-4,6) ; \\
\text { alta intensidade } \\
\text { (OR }=3,9 ; 2,1-7,2) ; \\
\text { muito alta intensidade } \\
(\mathrm{OR}=7,1 ; 3,7-13,4)\end{array}$ & 11 \\
\hline Bos et al. 24 & Holanda/2004 & Transversal & $\begin{array}{l}877 \text { homens e } \\
476 \text { mulheres; } \\
38 \% \text { perdas e } \\
\text { recusas }\end{array}$ & $\begin{array}{l}\text { Questionário } \\
\text { formulado } \\
\text { pelos autores da } \\
\text { pesquisa }\end{array}$ & $\begin{array}{c}\text { Bombeiros }(20,0) \\
\text { Trabalhadores de } \\
\text { escritório }(14,0)\end{array}$ & 6 meses & $\begin{array}{l}\text { Risco aumentado para } \\
\text { indivíduos que realizam } \\
\text { atividades com alta } \\
\text { demanda biomecânica } \\
(\mathrm{OR}=1,6 ; 1,2-2,1)\end{array}$ & 10 \\
\hline Chen et al. 6 & Taiwan/2004 & Transversal & $\begin{array}{c}1.242 \\
\text { (homens } 1.193 \\
\text { e 49mulheres); } \\
\text { média de } \\
\text { idade }=44,5 ; \\
8 \% \text { perdas e } \\
\text { recusas }\end{array}$ & $\begin{array}{c}\text { Nordic } \\
\text { Musculoskeletal } \\
\text { Questionnaire }\end{array}$ & Taxistas $(19,0)$ & 12 meses & $\begin{array}{c}\text { Dirigir por mais de } \\
10 \text { horas/dia }(\mathrm{OR}=3,1 ; \\
\text { 1,6-6,1); atividade de } \\
\text { lazer muito freqüente } \\
(\mathrm{OR}=1,9 ; 1,1-3,3) ; \\
\text { stress moderado/ } \\
\text { severo no trabalho } \\
(\mathrm{OR}=1,8 ; 1,1-3,0) ; \\
\text { baixa saúde mental } \\
(\mathrm{OR}=1,7 ; 1,3-2,5) ; \\
\text { e trabalho autônomo } \\
(\mathrm{OR}=1,6 ; 1,1-2,4)\end{array}$ & 12 \\
\hline Menzel et al. 36 & $\begin{array}{c}\text { Estados } \\
\text { Unidos/2004 }\end{array}$ & Transversal & $\begin{array}{l}113 \text { ( } 13 \text { homens } \\
\text { e } 100 \text { mulheres); } \\
\text { média de idade } \\
=42,0 ; 6,6 \% \\
\text { perdas e recusas }\end{array}$ & $\begin{array}{c}\text { Cornell } \\
\text { Musculoskeletal } \\
\text { Disconfort } \\
\text { Questionnaire }\end{array}$ & $\begin{array}{l}\text { Profissionais de } \\
\text { enfermagem } \\
\text { (sem dados) }\end{array}$ & 7 dias & $\begin{array}{l}\text { Interação das variáveis } \\
\text { tarefas de mais alto risco } \\
\text { por hora com carga } \\
\text { carregada }(B=0,290)\end{array}$ & 8 \\
\hline Wills et al. 23 & $\begin{array}{l}\text { Reino Unido/ } \\
2004\end{array}$ & Coorte & $\begin{array}{l}1.008 \text { (960 homens } \\
\text { e } 48 \text { mulheres); } \\
\text { média de idade } \\
=19,4 ; 70 \% \\
\text { perdas e } \\
\text { recusas } \\
\end{array}$ & $\begin{array}{l}\text { s Diagnóstico } \\
\text { médico } \\
\text { baseado em } 3 \\
\text { questionamentos: } \\
\text { dor no ou ao } \\
\text { redor do joelho; } \\
\text { dano não visível no } \\
\text { local; sem evidência } \\
\text { de trauma }\end{array}$ & $\begin{array}{l}\text { Recrutas do } \\
\text { exército }(8,7)\end{array}$ & Pontual & - & 7 \\
\hline
\end{tabular}

(continua) 
Tabela 1 (continuação)

\begin{tabular}{|c|c|c|c|c|c|c|c|c|}
\hline Referência & $\begin{array}{l}\text { Local do } \\
\text { estudo/Ano }\end{array}$ & Delineamento & Amostra & Instrumento & $\begin{array}{c}\text { Categoria de } \\
\text { trabalho e prevalência } \\
\text { de dor joelho (\%) }\end{array}$ & $\begin{array}{l}\text { Período } \\
\text { da } \\
\text { medida }\end{array}$ & $\begin{array}{l}\text { Fatores associados } \\
\text { (medidas de efeito; } \\
\text { IC95\%) }\end{array}$ & Escore * \\
\hline Gomez et al. 35 & $\begin{array}{c}\text { Estados } \\
\text { Unidos/2003 }\end{array}$ & Coorte & $\begin{array}{c}1.706(1.064 \\
\text { homens e } 642 \\
\text { mulheres); média } \\
\text { de idade }=45,1 ; \\
1,3 \% \text { perdas e } \\
\text { recusas }\end{array}$ & $\begin{array}{c}\text { Questionário } \\
\text { sobre incidência } \\
\text { de dor ou } \\
\text { desconforto }\end{array}$ & Agricultores $(29,0)$ & 12 meses & $\begin{array}{c}\text { Idade avançada (OR = } \\
\text { 1,2; } 1,1-1,3) ; \mathrm{IMC} \\
\text { obesidade }(\mathrm{OR}=1,4 ; \\
\text { 1,1-1,7); trabalho com } \\
\text { trator (OR = 1,5; } \\
\text { 1,2-1,9); e ordenha } \\
(\mathrm{OR}=1,4 ; 1,1-1,7)\end{array}$ & 11 \\
\hline $\begin{array}{l}\text { Gurgueira } \\
\text { et al. } 38\end{array}$ & Brasil/2003 & Transversal & $\begin{array}{l}105 \text { mulheres } \\
\text { auxiliares e } \\
\text { técnicos de } \\
\text { enfermagem; } \\
\text { 4,5\% perdas e } \\
\text { recusas }\end{array}$ & $\begin{array}{c}\text { Nordic } \\
\text { Musculoskeletal } \\
\text { Questionnaire }\end{array}$ & $\begin{array}{l}\text { Enfermeiras (anual: } \\
\text { 33,3; } 7 \text { dias: } 14,3 \text { ) }\end{array}$ & $\begin{array}{l}12 \text { meses e } \\
\text { nos últimos } \\
7 \text { dias }\end{array}$ & - & 8 \\
\hline Merlino et al. 4 & $\begin{array}{c}\text { Estados } \\
\text { Unidos/2003 }\end{array}$ & Transversal & $\begin{array}{c}996 \\
\text { (929 homens } \\
\text { e } 67 \text { mulheres); } \\
\text { média de idade } \\
=27,7 ; 15,2 \% \\
\text { perdas e } \\
\text { recusas }\end{array}$ & $\begin{array}{c}\text { Nordic } \\
\text { Musculoskeletal } \\
\text { Questionnaire } \\
\text { modificado }\end{array}$ & $\begin{array}{l}\text { Aprendizes de } \\
\text { construção }(38,4)\end{array}$ & 12 meses & $\begin{array}{c}\text { Mais de } 4 \text { anos de } \\
\text { trabalho no mercado } \\
\text { da construção (OR = } \\
2,0 ; 1,4-3,1) ; \text { posição } \\
\text { viciosa (OR = 1,3; 0,9-1,8); } \\
\text { posição estática } \\
\text { por longo tempo } \\
\text { (OR = 2,1; 1,5-2,9); } \\
\text { condição de trabalho } \\
\text { ruim }(\mathrm{OR}=1,5 ; 1,0-2,1)\end{array}$ & ; \\
\hline Smith et al. 40 & Japão/2003 & Transversal & $\begin{array}{l}247 \text { mulheres; } \\
\text { média de idade } \\
=33,4 ; 11,3 \% \\
\text { perdas e recusas }\end{array}$ & $\begin{array}{c}\text { Nordic } \\
\text { Musculoskeletal } \\
\text { Questionnaire } \\
\text { modificado }\end{array}$ & Enfermeiras $(23,5)$ & 12 meses & - & 8 \\
\hline $\begin{array}{l}\text { Goldsheyder } \\
\text { et al. } 34\end{array}$ & $\begin{array}{c}\text { Estados } \\
\text { Unidos/2002 }\end{array}$ & Transversal & $\begin{array}{l}312 \text { homens; } \\
\text { média de idade } \\
=39,9 ; 29,8 \% \\
\text { perdas e recusas }\end{array}$ & $\begin{array}{c}\text { lowa Construction } \\
\text { Questionnaire }\end{array}$ & $\begin{array}{c}\text { Construção } \\
\text { (12 meses: } 41,0 ; \\
7 \text { dias: } 22,0)\end{array}$ & $\begin{array}{l}12 \text { meses e } \\
\text { nos últimos } \\
7 \text { dias }\end{array}$ & - & 8 \\
\hline $\begin{array}{l}\text { Jensen \& } \\
\text { Kofoed } 21\end{array}$ & Dinamarca/2002 & Transversal & $\begin{array}{c}282 \text { homens; } \\
\text { média de idade } \\
=37,0 \text { nos instala- } \\
\text { dores e de } 22,0 \\
\text { anos nos aprendizes; } \\
8,8 \% \text { perdas e } \\
\text { recusas }\end{array}$ & $\begin{array}{l}\text { Nordic } \\
\text { Musculoskeletal } \\
\text { Questionnaire }\end{array}$ & $\begin{array}{c}\text { Instaladores de } \\
\text { piso (anual: } 56,0 \text {; } \\
7 \text { dias: } 30,0 \text { ); } \\
\text { aprendizes de } \\
\text { instaladores (anual: } \\
\text { 48,0; } 7 \text { dias: } 14,0 \text { ) }\end{array}$ & $\begin{array}{l}12 \text { meses e } \\
\text { nos últimos } \\
7 \text { dias }\end{array}$ & $\begin{array}{c}\text { Elevado esforço } \\
\text { físico }(\mathrm{OR}=9,1 ; \\
\text { 1,1-78,8); elevado } \\
\text { esforço psicológico } \\
\text { no trabalho } \\
(\mathrm{OR}=2,5 ; 1,0-6,0) \\
\text { e stress no trabalho } \\
(\mathrm{OR}=2,3 ; 1,0-5,2)\end{array}$ & 7 \\
\hline $\begin{array}{l}\text { Miranda } \\
\text { et al. } 15\end{array}$ & Finlândia/2002 & Coorte & $\begin{array}{c}2.122 \text { ( } 1.535 \\
\text { homens e } 586 \\
\text { mulheres); idade } \\
\text { (menos } 35 \text { anos: } \\
\text { 17\%; 35-44 anos: } \\
\text { 32\%; } 45-54 \text { anos: } \\
\text { 36\%; e mais } 55 \text { : } \\
\text { anos 15\%); média = } \\
45,3 \% ; 53 \% \\
\text { perdas e recusas }\end{array}$ & $\begin{array}{c}\text { Nordic } \\
\text { Musculoskeletal } \\
\text { Questionnaire } \\
\text { modificado }\end{array}$ & $\begin{array}{l}\text { Trabalhadores da } \\
\text { indústria }(10,0)\end{array}$ & $\begin{array}{l}\text { Incidência } \\
\text { de dor no } \\
\text { período de } \\
\text { um ano }\end{array}$ & $\begin{array}{c}\text { Sexo feminino } \\
(\mathrm{OR}=1,6 ; 1,0-2,4) ; \\
\text { sobrepeso }(\mathrm{OR}=1,9 ; \\
1,2-3,8) ; \text { tabagismo } \\
(\mathrm{OR}=1,3 ; 0,9-2,0) ; \\
\text { e ter tido lesão } \\
\text { anterior nos joelhos } \\
(\mathrm{OR}=2,7 ; 1,8-4,1)\end{array}$ & 11 \\
\hline
\end{tabular}

(continua) 


\begin{tabular}{|c|c|c|c|c|c|c|c|c|}
\hline Referência & $\begin{array}{l}\text { Local do } \\
\text { estudo/Ano }\end{array}$ & Delineamento & Amostra & Instrumento & $\begin{array}{c}\text { Categoria de } \\
\text { trabalho e prevalência } \\
\text { de dor joelho }(\%)\end{array}$ & $\begin{array}{c}\text { Período } \\
\text { da } \\
\text { medida }\end{array}$ & $\begin{array}{l}\text { Fatores associados } \\
\text { (medidas de efeito; } \\
\text { IC95\%) }\end{array}$ & Escore * \\
\hline Pinheiro et al. 22 & Brasil/2002 & Transversal & $\begin{array}{l}78 \text { ( } 45 \text { homens } \\
\text { e } 33 \text { mulheres); } \\
\text { média idade = } \\
39,0 \text { anos; sem } \\
\text { perdas e recusas }\end{array}$ & $\begin{array}{c}\text { Nordic } \\
\text { Musculoskeletal } \\
\text { Questionnaire } \\
\text { modificado }\end{array}$ & $\begin{array}{l}\text { Bancários (anual: } \\
28,9 ; 7 \text { dias: } 14,5 \text { ) }\end{array}$ & $\begin{array}{l}12 \text { meses e } \\
\text { nos últimos } \\
7 \text { dias }\end{array}$ & - & 7 \\
\hline Miranda et al. 30 & $\begin{array}{c}\text { Finlândia/ } \\
2001\end{array}$ & Transversal & $\begin{array}{c}3.312 \\
\text { (2.472 homens } \\
\text { e } 873 \text { mulheres); } \\
\text { média idade } \\
=45,3 \text { anos; } \\
23 \% \text { perdas } \\
\text { e recusas }\end{array}$ & $\begin{array}{c}\text { Nordic } \\
\text { Musculoskeletal } \\
\text { Questionnaire } \\
\text { modificado }\end{array}$ & $\begin{array}{l}\text { Trabalhadores } \\
\text { da indústria } \\
\text { (homens: } 24,0 ; \\
\text { mulheres: } 22,0 \text { ) }\end{array}$ & $\begin{array}{l}12 \text { meses e } \\
\text { nos últimos } \\
7 \text { dias }\end{array}$ & $\begin{array}{c}\text { Idade superior a } \\
55 \text { anos (OR }=2,4 ; \\
\text { 1,6-3,7); obesidade } \\
(\mathrm{OR}=2,0 ; 1,4-2,9) ; \\
\text { stress mental } \\
(\mathrm{OR}=3,5 ; 2,4-5,1) ; \\
\text { lesão nos joelhos } \\
\text { (OR = 3,1; 2,4-4,1); } \\
\text { grande risco de } \\
\text { acidentes no trabalho } \\
\text { devido a escorregar, } \\
\text { tropeçar ou cair de } \\
\text { escada (OR = 1,9; } \\
\text { 1,7-2,6); trabalho físico } \\
\text { muito estressante } \\
\text { (OR = 3,4; 1,8-6,2) }\end{array}$ & 12 \\
\hline Nahit et al. 31 & $\begin{array}{c}\text { Reino Unido/ } \\
2001\end{array}$ & Transversal & $\begin{array}{c}1.081 \\
\text { (734 homens e } \\
347 \text { mulheres); } \\
9 \% \text { perdas e } \\
\text { recusas }\end{array}$ & $\begin{array}{l}\text { Utilização de } \\
\text { uma figura para } \\
\text { indicar local } \\
\text { da dor }\end{array}$ & $\begin{array}{l}\text { Trabalhos diversos } \\
\text { (20,5; homens: } 23,8 ; \\
\text { mulheres: } 13,5) ; \\
\text { bombeiros }(24,0) ; \\
\text { comerciários }(14,0) ; \\
\text { construção naval }(7,0) ; \\
\text { dentistas }(13,0) ; \\
\text { infantaria exército }(38,0) ; \\
\text { oficiais do exército }(44,0) \\
\text { enfermeiras }(9,0) ; \\
\text { médicos }(16,0) ; \\
\text { trabalhadores dos } \\
\text { correios }(19,0) ; \\
\text { auxiliar de escritório } \\
\text { exército }(26,0) ; \\
\text { policiais }(14,0) ; \\
\text { indústria de } \\
\text { papel }(21,0)\end{array}$ & $\begin{array}{l}\text { Último } \\
\text { mês }\end{array}$ & $\begin{array}{c}\text { Levantar mais de } \\
11 \mathrm{~kg} \text { com uma o } \\
(\mathrm{OR}=1,3 ; 0,9-2,0) ; \\
\text { levantar maisde } 23 \mathrm{~kg} \\
\text { com duas mãos } \\
(\mathrm{OR}=1,2 ; 0,7-1,9) ; \\
\text { carregar mais de } 23 \mathrm{~kg} \\
\text { em um dos ombros } \\
(\mathrm{OR}=2,2 ; 01,3-3,7)\end{array}$ & 12 \\
\hline Jensen et al. 27 & $\begin{array}{c}\text { Dinamarca/ } \\
2000\end{array}$ & Transversal & $\begin{array}{l}966 \text { homens; } \\
\text { idade } 26-72 \text { anos; } \\
45 \% \text { perdas } \\
\text { e recusas }\end{array}$ & $\begin{array}{c}\text { Nordic } \\
\text { Musculoskeletal } \\
\text { Questionnaire }\end{array}$ & $\begin{array}{l}\text { Instaladores de } \\
\text { piso (anual: } 53,0 ; \\
7 \text { dias: } 32,4 \text { ); } \\
\text { Carpinteiros (anual: } \\
\text { 43,0; } 7 \text { dias: } 24,0 \text { ); } \\
\text { Designers gráficos } \\
\text { (anual: } 15,0 ; \\
7 \text { dias: } 7,0 \text { ) }\end{array}$ & $\begin{array}{l}12 \text { meses e } \\
\text { nos últimos } \\
7 \text { dias }\end{array}$ & $\begin{array}{c}\text { Trabalho como } \\
\text { carpinteiro } \\
(\mathrm{OR}=5,0 ; 3,5-7,4) \\
\text { trabalho como } \\
\text { colocador de piso } \\
(\mathrm{OR}=10,9 ; 6,3-19,5)\end{array}$ & 12 \\
\hline
\end{tabular}

(continua) 


\begin{tabular}{|c|c|c|c|c|c|c|c|c|}
\hline Referência & $\begin{array}{l}\text { Local do } \\
\text { estudo/Ano }\end{array}$ & Delineamento & Amostra & Instrumento & $\begin{array}{c}\text { Categoria de } \\
\text { trabalho e prevalência } \\
\text { de dor joelho }(\%)\end{array}$ & $\begin{array}{c}\text { Período } \\
\text { da } \\
\text { medida }\end{array}$ & $\begin{array}{l}\text { Fatores associados } \\
\text { (medidas de efeito; } \\
\text { IC95\%) }\end{array}$ & Escore * \\
\hline O'Reilly et al. 9 & $\begin{array}{c}\text { Reino } \\
\text { Unido/2000 }\end{array}$ & Transversal & $\begin{array}{c}4.057 \text { ( } 1.961 \\
\text { homens e } 2.096 \\
\text { mulheres); idade } \\
\text { entre } 40 \text { e } 79 \text { anos; } \\
\text { 18\% perdas e } \\
\text { recusas }\end{array}$ & $\begin{array}{l}\text { Questionário } \\
\text { auto-aplicado } \\
\text { (postado) } \\
\text { po } \\
\text { t }\end{array}$ & $\begin{array}{c}\text { Geral: } 28,0 ; \\
\text { (homens: } 28,0 ; \\
\text { mulheres: } 29,0) ; \\
\text { Homens: carpinteiros } \\
(60,9) \text {, mineiros }(45,1) \text {, } \\
\text { construção }(42,6), \\
\text { alfaiate }(35,3), \\
\text { motorista }(27,8), \\
\text { atendente }(25,0), \\
\text { supervisor }(22,0), \\
\text { policial/segurança }(23,8) \text {, } \\
\text { professor }(20,8) ; \\
\text { mulheres: assistente } \\
\text { de vendas }(37,1), \\
\text { supervisora }(20,4), \\
\text { trabalho em máquina } \\
\text { têxtil }(31,5), \\
\text { enfermeira }(31,6), \\
\text { recepcionista }(28,2), \\
\text { atendente }(24,5), \\
\text { secretária }(23,4), \\
\text { faxineira }(21,9), \\
\text { professora }(18,9)\end{array}$ & 12 meses & $\begin{array}{c}\text { Trabalhar como } \\
\text { carpinteiro } \\
(\mathrm{OR}=4,6 ; 1,9-11,1) ; \\
\text { mineiro }(\mathrm{OR}=1,9 ; \\
1,3-2,8) ; \text { construção } \\
(\mathrm{OR}=2,4 ; 1,4-4,1) ; \\
\text { ocupações envolvem } \\
\text { trabalho manual } \\
(\mathrm{OR}=1,8 ; 1,4-2,4) ; \\
\text { e montadores } \\
(\mathrm{OR}=1,6 ; 1,0-2,6)\end{array}$ & 7 \\
\hline Dimov et al. 32 & $\begin{array}{c}\text { Estados } \\
\text { Unidos/2000 }\end{array}$ & Transversal & $\begin{array}{l}73 \text { ( } 71 \text { homens } \\
\text { e } 2 \text { mulheres); } \\
\text { média de idade } \\
=37,6 \text { anos; } \\
\text { sd = } 13,45 \text { anos; } \\
\text { sem perdas e } \\
\text { recusas }\end{array}$ & $\begin{array}{c}\text { Body } \\
\text { Segment } \\
\text { Instrument } \\
\text { (escala modifi- } \\
\text { cada de } \\
\text { Bishop-Corlett) }\end{array}$ & Carpinteiros $(45,2)$ & 12 meses & $\begin{array}{l}\text { A maior prevalência } \\
\text { de dor encontrada } \\
\text { (nas costas) não } \\
\text { foi diferente } \\
\text { da prevalência } \\
\text { de gonalgia }\end{array}$ & 8 \\
\hline $\begin{array}{l}\text { Lagerstrom } \\
\text { et al. } 29\end{array}$ & Suécia/1995 & Transversal & $\begin{array}{l}688 \text { mulheres; } \\
\text { 36\% perdas e } \\
\text { recusas }\end{array}$ & $\begin{array}{l}\text { Nordic } \\
\text { Musculoskeletal } \\
\text { Questionnaire. } \\
\text { Para cada sintoma, } \\
\text { uma escala de } \\
\text { pontos de } 0 \text { a } 9 \text { foi } \\
\text { utilizada para deter- } \\
\text { minar a gravidade } \\
\text { dos sintomas } \\
\text { (6 ou mais pontos } \\
\text { = dor severa) }\end{array}$ & $\begin{array}{l}\text { Enfermeiras }(30,0) \\
\text { oi } \\
\text { s- } \\
\text { e }\end{array}$ & 12 meses & $\begin{array}{c}\text { Idade }(\mathrm{OR}=1,3 ; \\
1,1-1,5) ; \text { para dor } \\
\text { severa: idade }(1,5 ; \\
1,1-1,9) ; \text { e elevado } \\
\text { IMC }(\mathrm{OR}=3,2 ; \\
1,7-5,9)\end{array}$ & 11 \\
\hline Hunting et. al. 20 & $\begin{array}{c}\text { Estados } \\
\text { Unidos/1994 }\end{array}$ & Transversal & $\begin{array}{l}302 \text { homens } \\
\text { e } 6 \text { mulheres; } \\
23 \% \text { perdas e } \\
\text { recusas }\end{array}$ & $\begin{array}{l}\text { Cumulative } \\
\text { Trauma } \\
\text { Disorders } \\
\text { Surveillance } \\
\text { Questionnaire } \\
\text { adaptado }\end{array}$ & $\begin{array}{c}\text { Eletricista (DEF1: } \\
\text { 33,0 DEF1; } \\
\text { DEF2: 16,0; } \\
\text { DEF3: 26,0) ** }\end{array}$ & 12 meses & $\begin{array}{l}\text { Trabalhar mais de } \\
10 \text { anos como } \\
\text { eletricista }(\mathrm{OR}=2,8)\end{array}$ & 7 \\
\hline
\end{tabular}

(continua) 


\begin{tabular}{|c|c|c|c|c|c|c|c|c|}
\hline Referência & $\begin{array}{l}\text { Local do } \\
\text { estudo/Ano }\end{array}$ & Delineamento & Amostra & Instrumento & $\begin{array}{c}\text { Categoria de } \\
\text { trabalho e prevalência } \\
\text { de dor joelho }(\%)\end{array}$ & $\begin{array}{l}\text { Período } \\
\text { da } \\
\text { medida }\end{array}$ & $\begin{array}{l}\text { Fatores associados } \\
\text { (medidas de efeito; } \\
\text { IC95\%) }\end{array}$ & Escore * \\
\hline $\begin{array}{l}\text { Holmstrom } \\
\text { et al. } 25\end{array}$ & Suécia/1992 & Transversal & $\begin{array}{l}1.772 \text { homens; } \\
25 \% \text { perdas e } \\
\text { recusas }\end{array}$ & $\begin{array}{l}\text { Baseado no } \\
\text { questionamento: } \\
\text { dor ou desconforto } \\
\text { no local, experien- } \\
\text { ciado algumas } \\
\text { vezes, freqüente- } \\
\text { mente ou muito } \\
\text { freqüentemente nos } \\
\text { últimos } 12 \text { meses }\end{array}$ & 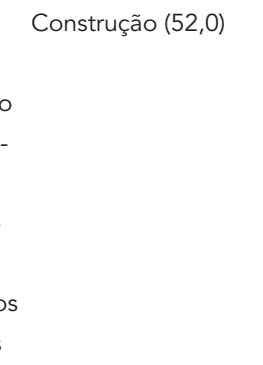 & 12 meses & - & 11 \\
\hline $\begin{array}{l}\text { Kivimaki } \\
\text { et al. } 28\end{array}$ & $\begin{array}{c}\text { Finlândia/ } \\
1992\end{array}$ & Transversal & $\begin{array}{l}312 \text { homens; } \\
\text { idade } 25 \text { a } 49 \\
\text { anos; } 28 \% \\
\text { perdas e } \\
\text { recusas }\end{array}$ & $\begin{array}{l}\text { Questionamento } \\
\text { auto-aplicado } \\
\text { sobre dor em algum } \\
\text { dos joelhos ao } \\
\text { realizar alguma } \\
\text { dessas tarefas: } \\
\text { subir escadas, } \\
\text { descer escadas, } \\
\text { caminhar no plano, } \\
\text { permanecer sentado, } \\
\text { descansar à noite, } \\
\text { agachar e ajoelhar }\end{array}$ & $\begin{array}{l}\text { Instaladores } \\
\text { de carpete } \\
\text { e piso }(74,0) \text {, } \\
\text { Pintores }(61,0)\end{array}$ & $\begin{array}{l}\text { No último } \\
\text { mês }\end{array}$ & $\begin{array}{l}\text { Caminhar no plano, } \\
\text { trabalhar ajoelhado, } \\
\text { permanecer sentado } \\
\text { e descansar à noite }\end{array}$ & 10 \\
\hline Chen et al. 39 & China/1991 & Transversal & $\begin{array}{c}463 \text { homens; } \\
\text { sem informação } \\
\text { perdas e } \\
\text { recusas }\end{array}$ & $\begin{array}{l}\text { Avaliação médica } \\
\text { e questionário } \\
\text { auto-aplicado } \\
\text { sobre dor }\end{array}$ & $\begin{array}{l}\text { Trabalhadores } \\
\text { comércio: baixa } \\
\text { temperatura } \\
\left(-10 \text { a }-25^{\circ} \mathrm{C}: 46,6\right) ; \\
\text { lojas gelo } \\
\left(-5 \text { a }+5^{\circ} \mathrm{C}: 50,8\right) ; \\
\text { comérciocomum } \\
\left(20 \text { a } 30^{\circ} \mathrm{C}: 14,5\right)\end{array}$ & 12 meses & - & 10 \\
\hline
\end{tabular}

* Escore de qualidade de acordo com Downs \& Black 18;

** DEF1: dor no último ano por pelo menos 3 vezes ou dor que durou mais que 1 semana/ano; DEF2: dor no último ano pelo menos 1 vez ao mês ou durou mais que 1 semana/ano, sem história de lesão traumática prévia; DEF3: dor no último ano por pelo menos 1 vez ao mês ou que durou mais que 1 semana/ano, com história de lesão aguda.

1,52-2,93) e trabalho em condições inadequadas (OR = 1,47; IC95\%: 1,04-2,07).

O aumento da idade, do IMC 29 e a interação entre horas de trabalho e carga transportada 36 foram os fatores de risco associados à gonalgia entre os trabalhadores de enfermagem. Esses estudos foram, em sua maioria, realizados com mulheres.

As prevalências de gonalgia entre os instaladores de piso descritas em quatro diferentes estudos foram de 53,0, 56,0, 65,0 e 74,0\% 21,26,27,28. Dois desses estudos realizaram somente análise bruta para verificação de associação entre o desfecho e as variáveis preditoras 21,28 . Nesses, as variáveis "elevado esforço físico no trabalho", "ele- vado esforço psicológico no trabalho", "stress no trabalho", "trabalho ajoelhado", "trabalho sentado" e "caminhar no plano" estiveram associadas com gonalgia. Utilizando análise para múltiplos preditores, Jensen 26 encontrou um odds ratio de gonalgia de 7,1 (IC95\%: 3,70-13,40) entre os trabalhadores expostos à elevadíssima intensidade de trabalho ajoelhado e agachado, quando comparados a trabalhadores não expostos a estas posições. Em outro estudo, Jensen et al. 27 encontraram que colocadores de piso tiveram um odds ratio de gonalgia de 10,9 (IC95\%: 6,26-19,50) quando comparados a designers gráficos. 


\section{Discussão}

A gonalgia é um problema de saúde comum no mundo ${ }^{6}$. Sua ocorrência na população é elevada 42,43 , sendo que, entre trabalhadores, sua prevalência/incidência atinge níveis preocupantes 25,27,28. Apesar disso, ainda não são muitos os estudos que associam gonalgia à ocupação e fatores ocupacionais. Nesta revisão encontramos que $34,6 \%$ dos estudos somente descreviam a prevalência de dor nas ocupações sem investigar sua relação com fatores ocupacionais. O livro Musculoskeletal Disorders and Workplace Factors do $\mathrm{NIOSH}^{3}$, considerado como uma das grandes referências sobre problemas musculoesqueléticos e trabalho, não aborda os problemas de joelho em sua revisão.

Em termos metodológicos os artigos avaliados tiverem escore baixo, com quase metade deles obtendo pontuação inferior a oito de um total de treze. Estudos com trabalhadores, freqüentemente, têm grande número de perdas e recusas apresentando, portanto, limitações relativas à representatividade amostral. Muitas vezes trabalhadores podem recusar-se a responder questionários ou participar de exames clínicos com receio de demissão caso seja identificado um problema de saúde.

Apesar de ter sido criado há quase duas décadas, o Questionário Nórdico para Análise de Sintomas Musculoesqueléticos foi o instrumento mais utilizado para a avaliação de gonalgia. Isso provavelmente se deve ao fato desse instrumento ser de fácil compreensão e aplicação, e de apresentar bons índices de confiabilidade 22 . $\mathrm{O}$ instrumento tem como principal limitação a falta de uma medida de gravidade de sintomas baseada na freqüência e/ou na intensidade dos mesmos 22 .

A sumarização dos resultados dos 26 estudos foi bastante dificultada pela grande variação de objetivos e desfechos em cada estudo. Apesar das diferenças metodológicas, as prevalências nos últimos doze meses ficaram na faixa de 16 a $51 \%$ (percentis entre 10 e 90). Os estudos de Jensen \& Kofoed 21, Jensen 26, Jensen et al. 27 e Holstrom et al. ${ }^{25}$, que encontraram prevalências acima desse valor tinham a população composta somente por homens. O estudo de Fassa et al. 19 encontrou prevalências de 11,2 e 12,1\%, respectivamente, para crianças trabalhadoras no comércio e na construção. Já os estudos de Chen et al. 39 e Jensen et al. 27 encontraram prevalências de gonalgia inferiores a $16,0 \%$ em trabalhadores do comércio e designers gráficos.

Cabe salientar que era esperada a variabilidade da prevalência nas diferentes ocupações, uma vez que, cada categoria ocupacional apresenta riscos ergonômicos específicos, bem como, um perfil relativo a sexo, idade, escolaridade e renda. No caso das gonalgias, as principais exposições que geram estas diferenças são o esforço físico e as rotinas posturais de cada ocupação. Assim, foram observadas prevalências mais elevadas para os colocadores de piso e menores para crianças trabalhadoras no comércio, por exemplo.

As associações entre gonalgia e variáveis sócio-demográficas relatadas evidenciaram maior prevalência entre as mulheres, nos indivíduos mais velhos e com IMC elevado. Alguns estudos epidemiológicos defendem a idéia de que mulheres reportam mais dor do que homens 3,24 . Sendo a premissa verdadeira, corre-se o risco de viés de informação, mas existe certa plausibilidade nessa prevalência mais elevada entre as mulheres, uma vez que, atualmente elas estão mais inseridas no mercado de trabalho e têm exposição freqüente a cargas ergonômicas ${ }^{44}$. Além disso, muitas mulheres sofrem o efeito da dupla jornada de trabalho, a remunerada e a doméstica.

$\mathrm{O}$ risco linear de aumento da gonalgia com idade pode dever-se ao fato de que os processos degenerativos, de um modo geral, podem estar bem avançados, trazendo como conseqüências o desgaste das estrutura osteomusculares e orgânicas, associado a cargas de trabalho semelhantes a dos mais jovens 45 . A "carga extra" que a estrutura osteo-musculo-articular é obrigada a sustentar aumenta o risco de gonalgia em pessoas com sobrepeso e obesas.

O trabalho na posição ajoelhada, o estresse físico e psicológico e o carregamento de peso foram os fatores ocupacionais que apareceram associados à gonalgia em mais de um estudo. Segundo Kivimaki et al. 28, em algumas ocupações a proporção de trabalho ajoelhado é muito elevada e isto causaria prolongado estresse sobre a articulação patelar, levando à dor. Demandas físicas no trabalho, onde está inserido o carregamento de peso, apresentam forte associação com dor em outras articulações, tais como pescoço, ombros, cotovelos e punhos ${ }^{3}$. Muitos estudos têm identificado variáveis psicossociais, como autopercepção de estresse no trabalho, insatisfação no trabalho e saúde mental como importantes determinantes de gonalgia, tanto em nível ocupacional como populacional 8,46,47.

Menos da metade dos estudos revisados realizou controle para fatores de confusão. Embora os aspectos descritivos sejam relevantes, esta abordagem limita-se à identificação de fatores de risco, uma vez que as associações encontradas podem ser fruto de outros fatores não avaliados simultaneamente. Entre os estudos que examinaram associações, somente um utilizou como medida de efeito a razão de prevalências. A pre- 
valência de gonalgia em trabalhadores em todos os estudos foi maior do que $10 \%$, deste modo, a utilização de odds ratio superestima os riscos encontrados 48,49.

Um dos grandes problemas do delineamento transversal é a causalidade reversa, que não permite estabelecer se a exposição precedeu o desfecho ou vice-versa ${ }^{50}$. Nesse caso, trabalhadores com substancial gonalgia podem ter deixado de trabalhar em ocupações que apresentam alta demanda física nesta articulação, diminuindo a estimativa da ocorrência desta no grupo estudado 26. Essa relação poderia ser melhor compreendida se variáveis relativas ao tempo de desempenho daquela função fossem incluídas.

\section{Conclusões}

O presente estudo mostrou que a estimativa de ocorrência de gonalgia, apesar de apresentar grande variabilidade, foi elevada entre as ocupações pesquisadas. Sexo feminino, idade avançada, IMC elevado, trabalhar ajoelhado e carregar peso no trabalho foram as variáveis associadas à gonalgia mais recorrentes nos estudos.

Apesar disso, dentre os problemas musculoesqueléticos encontrados em trabalhadores, a dor no joelho ainda é pouco estudada. Esta re- visão tenta preencher uma lacuna importante da epidemiologia ocupacional relacionada aos problemas musculoesqueléticos, dando suporte à produção de novos conhecimentos sobre o assunto. A realização de estudos longitudinais, que permitam estabelecer a temporalidade dos acontecimentos, que utilizem grupo de comparação, com tamanho amostral adequado para estudos de associação e uma avaliação minuciosa das exposições ocupacionais referentes a cada tipo de profissão são fundamentais para a condução de estudos válidos, precisos e abrangentes sobre o tema.

Iniciativas que busquem soluções no campo da ergonomia, divulgação de estratégias para prevenir/remediar o problema, bem como o desenvolvimento de políticas públicas que minimizem o impacto das exposições ocupacionais sobre a articulação dos joelhos são necessárias para diminuir o impacto que esta morbidade tem sobre trabalhadores de diversas ocupações. Utilização de protetores de joelhos, intervalos controlados de descanso com realização de alongamentos da musculatura de membros inferiores e adequação das cargas de trabalho a serem transportadas conforme a idade, sexo e estrutura física são algumas das alternativas que podem ajudar a minimizar esse tipo de problema entre os trabalhadores.

\section{Resumo}

Com o objetivo de reunir informações sobre gonalgia entre trabalhadores e fatores ocupacionais associados, foi realizada uma revisão sistemática nas bases de dados MEDLINE, LILACS, SciELO, Free Medical Journals, entre outros, referentes ao período 1990-2006, usando-se os descritores gonalgia, knee, knee pain, knee joint, knee dislocation, knee injuries, work, workplace, workload, employment, occupations, industry, occupational, workers, arthrosis, osteoarthritis e seus equivalentes em português e espanhol. Dos 2.263 estudos inicialmente encontrados, somente 26 cumpriram os critérios necessários para permanecer na revisão. As prevalências de gonalgia nos últimos 12 meses variaram entre $11,2 \%$ e 60,9\%, e os principais fatores associados foram: sexo feminino, idade avançada, índice de massa corporal elevado, trabalhar ajoelhado e carregar peso no trabalho.

Doenças Muscolosqueléticas; Saúde do Trabalhador; Literatura de Revisão

\section{Colaboradores}

M. C. Silva participou da elaboração do projeto, revisão da bibliografia, avaliação por pontos dos artigos que permaneceram na revisão e redação final do artigo. A. G. Fassa contribuiu na elaboração do projeto e redação final do artigo. M. R. Domingues realizou a avaliação dos artigos que permaneceram na revisão e redação final do artigo. D. Kriebel participou na elaboração do projeto e redação final do artigo.

\section{Agradecimentos}

Este trabalho teve o apoio financeiro da Coordenação de Aperfeiçoamento de Pessoal de Nível Superior e do John E. Fogarty International Center of the National Institutes of Health. 


\section{Referências}

1. National Research Council and Institute Medicine. Musculoskeletal disorders and the workplace: low back and upper extremities. Washington DC: National Academies Press; 2001.

2. March LM, Bachmeier CMJ. Economics of osteoarthritis: a global perspective. Baillieres Clin Rheumatol 1997; 11:817-34.

3. National Institute for Occupational Safety and Health. Musculoskeletal disorders and workplace factors. Cincinnati: National Institute for Occupational Safety and Health; 1997.

4. Merlino LA, Rosecrance JC, Anton D, Cook TM. Symptoms of musculoskeletal disorders among apprentice construction workers. Appl Occup Environ Hyg 2003; 18:57-64.

5. Knoplich J. Musculoskeletal system: spinal column. In: Mende R, editor. Work pathology. Rio de Janeiro: Atheneu; 1995. p. 213-27.

6. Chen JC, Dennerlein JT, Shih TS, Chen CJ, Cheng Y, Chang WP. Knee pain and driving duration: a secondary analysis of the Taxi Drivers' Health Study. Am J Public Health 2004; 94:575-81.

7. American Academy of Orthopaedic Surgeons. 6 million a year seek medical care for knees. Rosemont: American Academy of Orthopaedic Surgeons; 1997.

8. Bergenudd H, Nilsson B, Lindgarde F. Knee pain in middle age and its relationship to occupational work load and psychosocial factors. Clin Orthop Relat Res 1989; (245):210-5.

9. O'Reilly SC, Muir KR, Doherty M. Occupation and knee pain: a community study. Osteoarthr Cartil 2000; 8:78-81.

10. Faucher M, Poiraudeau S, Lefevre-Colau MM, Rannou F, Fermanian J, Revel M. Assessment of the test-retest reliability and construct validity of a modified Lequesne index in knee osteoarthritis. Joint Bone Spine 2003; 70:520-5.

11. Felson DT, Naimark A, Anderson J, Kazis LE, Castelli W, Meenan RF. The prevalence of knee osteoarthritis in the elderly. The Framingham Osteoarthritis Study. Arthritis Rheum 1987; 30:914-8.

12. Jensen LK, Mikkelsen S, Loft IP, Eenberg W, Bergmann I, Logager V. Radiographic knee osteoarthritis in floorlayers and carpenters. Scand J Work Environ Health 2000; 26:257-62.

13. Hannan MT, Anderson JJ, Pincus T, Felson DT. Educational attainment and osteoarthritis: differential associations with radiographic changes and symptom reporting. J Clin Epidemiol 1992; 47:13947.

14. Lachance L, Sowers M, Jamadar D, Jannausch M, Hochberg M, Crutchfield M. The experience of pain and emergent osteoarthritis of the knee. Osteoarthr Cartil 2001; 9:527-32.

15. Miranda H, Viikari-Juntura E, Martikainen R, Riihimaki H. A prospective study on knee pain and its risk factors. Osteoarthr Cartil 2002; 10:623-30.

16. Andersen RE, Crespo CJ, Ling SM, Bathon JM, Bartlett SJ. Correlates of knee pain among US adults with and without radiographic knee osteoarthritis. J Rheumatol 1999; 47:1435-8.
17. Hannan MT, Felson DT, Pincus T. Analysis of the discordance between radiographic changes and knee pain in osteoarthritis of the knee. J Rheumatol 2000; 27:1513-7.

18. Downs HS, Black N. The feasibility of creating a checklist for the assessment of the methodological quality both of randomised and non-randomised studies of health care interventions. J Epidemiol Community Health 1998; 52:377-84.

19. Fassa AG, Facchini LA, Dall'Agnol MM, Christiani DC. Child labor and musculoskeletal disorders: The Pelotas (Brazil) epidemiologic survey. Public Health Rep 2005; 120:665-73.

20. Hunting KL, Welch LS, Cuccherini BA, Seiger LA. Musculoskeletal symptoms among electricians. Am J Ind Med 1994; 25:149-63.

21. Jensen LK, Kofoed LB. Musculoskeletal disorders among floor layers: is prevention possible? Appl Occup Environ Hyg 2002; 17:797-806.

22. Pinheiro FA, Trocoli BT, Carvalho CV. Validação do questionário nórdico de sintomas osteomusculares como medida de morbidade. Rev Saúde Pública 2002; 36:307-12.

23. Wills AK, Ramasamy A, Ewins DJ, Etherington J. The incidence and occupational outcome of overuse anterior knee pain during army recruit training. J R Army Med Corps 2004; 150:264-9.

24. Bos J, Mol E, Visser B, Frings-Dresen M. Risk of health complaints and disabilities among Dutch firefighters. Int Arch Occup Environ Health 2004; 77:373-82.

25. Holmstrom EB, Lindell J, Moritz U. Low back and neck/shoulder pain in construction workers: occupational workload and psychosocial risk factors. Part 1: relationship to low back pain. Spine 1992; 17:663-71.

26. Jensen LK. Knee-straining work activities, self-report knee disorders and radiographically determined knee osteoarthritis. Scand J Work Environ Health 2005; 31:68-74

27. Jensen LK, Mikkelsen S, Loft IP, Eenberg W. Workrelated knee disorders in floor layers and carpenters. J Occup Environ Med 2000; 42:835-42.

28. Kivimaki J, Riihimaki H, Hanninen K. Knee disorders in carpet and floor layers and painters. Scand J Work Environ Health 1992; 18:310-6.

29. Lagerstrom M, Wenemark M, Hagberg M, Hjelm EW. Occupational and individual factors related to musculoskeletal symptoms in five body regions among Swedish nursing personnel. Int Arch Occup Environ Health 1995; 68:27-35.

30. Miranda H, Viikari-Juntura E, Martikainen R, Takala EP, Riihimaki H. Physical exercise and musculoskeletal pain among forest industry workers. Scand J Med Sci Sports 2001; 11:239-46.

31. Nahit ES, MacFarlane GJ, Pritchard CM, Cherry NM, Silman AJ. Short term influence of mechanical factors on regional musculoskeletal pain: a study of new workers from 12 occupational groups. Occup Environ Med 2001; 58:374-81. 
32. Dimov M, Bhattacharya A, Lemasters G, Atterbury M, Greathouse L, Ollila-Glenn N. Exertion and body discomfort perceived symptoms associated with carpentry tasks: an on-site evaluation. AIHAJ 2000; 61:685-91.

33. Forde MS, Punnett L, Wegman DH. Prevalence of musculoskeletal disorders in union ironworkers. J Occup Environ Hyg 2005; 2:203-12.

34. Goldsheyder D, Nordin M, Weiner SS, Hiebert R. Musculoskeletal symptom survey among Mason tenders. Am J Ind Med 2002; 42:384-96.

35. Gomez MI, Hwang S, Stark AD, May JJ, Hallman EM, Pantea CI. An analysis of self-reported joint pain among New York farmers. J Agric Saf Health 2003; 9:143-57.

36. Menzel NN, Brooks SM, Bernard TE, Nelson A. The physical workload of nursing personnel: association with musculoskeletal discomfort. Int J Nurs Stud 2004; 41:859-67.

37. Da Silva MC, Fassa AG, Kriebel D. Musculoskeletal pain among ragpickers in a Southern city in Brazil. Am J Ind Med 2006; 28:327-36.

38. Gurgueira GP, Alexandre NMC, Corrêa Filho HR. Prevalência de sintomas musculo-esqueléticos em trabalhadoras de enfermagem. Rev Latinoam Enfermagem 2003; 11:608-13.

39. Chen F, Li T, Huang H, Holmer I. A field study of cold effects among cold store workers in China. Arctic Med Res 1991; 50 Suppl 6:99-103.

40. Smith DR, Kondo N, Tanaka E, Tanaka H, Hirasawa K,Yamagata Z. Musculoskeletal disorders among hospital nurses in rural Japan. Rural Remote Health 2003; 3:241.

41. Kuorinka I, Johnsson B, Viterberg H, BieringSorensen F, Andersson GB. Standardized nordic questionnaires for the analysis of musculoskeletal symptoms. Appl Ergon 1987; 18:233-37.
42. Suka M,Yoshida K. Musculoskeletal pain in Japan: prevalence and interference with daily activities. Mod Rheumatol 2005; 15:41-7.

43. Zeng Q, Zang C, Li X, Dong H, Zhang A, Lin L. Associated risk factors of knee osteoarthritis: a population survey in Taiyuan, China. Chin Med J 2006; 119:1522-7.

44. Santana V, Amorin AM, Oliveira R, Xavier S, Iriart J, Belitardo L. Housemaids and non-fatal occupational injuries. Rev Saúde Pública 2003; 37:65-74.

45. Moskowitz RW, Kelly MA, Lewallen DG. Understanding osteoarthritis of the knee-causes and effects. Am J Orthop 2004; 33(2 Suppl):5-9.

46. Nahit ES, Pritchard CM, Cherry NM, Silman AJ, MacFarlane GJ. The influence of work-related psychosocial factors and psychosocial distress on original musculoskeletal pain: a study of newly employed workers. J Rheumatol 2001; 28:1378-84.

47. O’Reilly KP, Muir KR, Doherty M. Knee pain and disability in the Nottingham community: association with poor health status and psychological distress. Br J Rheumatol 1998; 37:870-3.

48. Barros AJD, Hirakata VN. Alternatives for logistic regression in cross-sectional studies: an empirical comparison of models that directly estimate the prevalence ratio. BMC Med Res Methodol 2003; $3: 21$.

49. Thompson ML, Myers JE, Kriebel D. Prevalence odds ratio or prevalence ratio in the analysis of cross sectional data: what is to be done? Occup Environ Med 1998; 54:272-7.

50. Checkoway H, Pearce N, Kriebel D. Research methods in occupational epidemiology. New York: Oxford University Press; 2004.

Recebido em 23/Out/2006

Versão final reapresentada em 01/Fev/2007

Aprovado em 16/Abr/2007 